\title{
Incidence de la fermeture et de la réouverture des écoles sur la transmission de la COVID-19
}

\author{
Maryem El Jaouhari', Rojiemiahd Edjoc ${ }^{1 *}$, Lisa Waddell2, Patricia Huston ${ }^{3}$, Nicole Atchessi ${ }^{1}$, \\ Megan Striha ${ }^{1}$, Samuel Bonti-Ankomah ${ }^{1}$
}

\section{Résumé}

Contexte : À l'échelle mondiale, l'éducation des élèves des écoles primaires et secondaires a été gravement perturbée par la fermeture des écoles pour réduire la propagation de la maladie à coronavirus en 2019 (COVID-19). L'efficacité de la fermeture des écoles pour la réduction de la transmission de la COVID-19 et l'impact de la réouverture des écoles ne sont pas clairs.

Méthodes : Les critères de recherche pour cet examen rapide comprenaient des études empiriques, publiées ou prépubliées dans le monde entier avant le 25 janvier 2021, qui évaluaient l'efficacité de la fermeture des écoles pour réduire la propagation de la COVID-19 et l'incidence de la réouverture des écoles sur la transmission de la COVID-19.

Résultats : Vingt-quatre études sur l'incidence de la fermeture et de la réouverture des écoles sur la transmission de la COVID-19 ont été relevées dans les sept bases de données qui ont fait l'objet d'une recherche. Dans l'ensemble, les données probantes de ces études étaient mitigées et variées en raison de plusieurs facteurs, comme le moment de la mise en œuvre des mesures de santé publique, la conception de la recherche des études incluses et la variabilité entre les niveaux de scolarité examinés.

Conclusion : Les résultats préliminaires suggèrent que la fermeture des écoles ont une incidence limitée sur la réduction de la transmission de la COVID-19, d'autres interventions non pharmaceutiques étant considérées comme beaucoup plus efficaces. Toutefois, en raison des limites des études, d'autres recherches sont nécessaires pour appuyer l'utilisation de cette mesure de santé publique en réponse à la pandémie de COVID-19.
Cette oeuvre est mise à la disposition selon les termes de la licence internationale Creative Commons Attribution 4.0

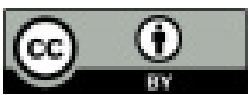

Affiliations

${ }^{1}$ Direction générale de la sécurité sanitaire et des opérations régionales, Agence de la santé publique du Canada, Ottawa, ON

${ }^{2}$ Laboratoire national de microbiologie, Agence de la santé publique du Canada, Winnipeg, MB

${ }^{3}$ Bureau de la conseillère scientifique en chef, Agence de la santé publique du Canada, Ottawa, ON

\section{*Correspondance : \\ rojiemiahd.edjoc@phac-aspc. gc.ca}

Citation proposée : El Jaouhari M, Edjoc R, Waddell L, Huston P, Atchessi N, Striha M, Bonti-Ankomah S. Incidence de la fermeture et de la réouverture des écoles sur la transmission de la COVID-19. Relevé des maladies transmissibles au Canada 2021;47(12):572-81. https://doi.org/10.14745/ccdr.v47i12a02f

Mots-clés : COVID-19, SRAS-CoV-2, fermeture des écoles, réouverture des écoles, intervention non pharmaceutique

\section{Introduction}

En date du 11 mars 2020, l'Organisation mondiale de la Santé a déclaré l'éclosion de maladie à coronavirus 2019 (COVID-19) en tant que pandémie (1). À l'échelle mondiale, les administrations ont commencé à mettre en œuvre diverses interventions non pharmaceutiques pour limiter la propagation et l'incidence de la COVID-19 causée par le coronavirus du syndrome respiratoire aigu sévère 2 (SRAS-CoV-2). Les écoles de fermeture étaient I'une des interventions non pharmaceutiques mis en œuvre; cependant, ces fermetures ont non seulement perturbé l'éducation et la routine quotidienne des élèves, mais aussi la vie des enseignants et des parents.
Même si la fermeture des écoles a été mise en œuvre pour lutter contre la propagation de COVID-19, elles étaient également associées à des effets négatifs sur la santé mentale et les progrès scolaires des élèves et entraînent une augmentation du stress chez les parents et les enseignants (2). En raison du manque d'interactions entre les pairs à l'école et de routines quotidiennes, on a signalé que les élèves ressentent de plus en plus de détresse, de solitude, d'anxiété et de symptômes dépressifs (2,3). Les routines scolaires sont essentielles au maintien du bien-être des élèves, en particulier ceux qui ont des besoins en santé mentale ou en éducation spécialisée (4). De plus, la fermeture des écoles a été associée à une réduction du rendement scolaire en raison du retard dans le progrès 
scolaire $(3,5,6)$. Il est difficile de savoir si l'apprentissage virtuel est aussi efficace et de nombreux étudiants des ménages à faible revenu ont de la difficulté à accéder, et à s'adapter au matériel en ligne (6).

Compte tenu des répercussions négatives de la fermeture des écoles, il est important de déterminer si elle est efficace dans la réduction significative de l'incidence de la COVID-19. Au départ, on a supposé que la fermeture des écoles serait efficace pour atténuer la propagation de la COVID-19 en fonction des données probantes sur la grippe saisonnière et épidémique $(7,8)$. En revanche, des études de modélisation menées en Ontario et dans l'ensemble du Canada au cours de la première et de la deuxième vague ont révélé que les fermetures des écoles avaient un impact limité sur la réduction de la transmission de la COVID-19 comparativement aux autres interventions non pharmaceutiques (9-11). D'autres études de modélisation ont fait état d'effets modestes de la fermeture des écoles en retardant le nombre de cas les plus élevés au début de la pandémie $(12,13)$, tandis que certaines études ont montré une plus petite ampleur de l'effet comparativement à d'autres interventions non pharmaceutiques $(14,15)$. Les premières études de modélisation reposaient sur I'hypothèse sous-jacente qu'il y a un faible risque de transmission chez les enfants. Même si les études de modélisation sont excellentes pour faire des prédictions éclairées, leur exactitude dépend des hypothèses et de la qualité des données utilisées. Dans l'ensemble, il était nécessaire d'évaluer les répercussions possibles de la fermeture d'écoles pour réduire la propagation de la COVID-19.

Cet examen résume les études empiriques sur l'efficacité des fermetures d'écoles et l'incidence de la réouverture des écoles sur la réduction de la transmission communautaire de COVID-19 et la diminution de l'incidence de COVID-19 dans les écoles primaires et secondaires. L'objet principal de cet article était I'incidence de la fermeture des écoles primaires et secondaires, quoique si les études comprenaient également des données d'autres types d'école, celles-ci ont également été incluses.

\section{Méthodes}

Nos critères de recherche comprenaient des études empiriques qui évaluaient l'incidence de la fermeture ou de la réouverture des écoles sur la COVID-19 qui ont été publiées avant le 25 janvier 2021. Les études de modélisation prédictive ont été exclues. Des recherches visant à récupérer les articles pertinents ont été menées dans PubMed, Scopus, BioXiv, MedRxiv, ArXiv, SSRN et Research Square, par le Groupe des sciences émergentes de l'Agence de la santé publique du Canada. Les termes de recherche comprenaient les suivants : école ET fermeture OU réouverture dans une base de données de la littérature COVID-19 mise à jour quotidiennement. Des références ont également été utilisées pour rechercher d'autres études pertinentes. La littérature incluse se limitait aux langues française et anglaise. Les articles $(n=966)$ ont ensuite fait l'objet d'une évaluation de la pertinence. Au total, cinq études d'observation et dix-neuf études écologiques ont été jugées pertinentes (voir les tableaux A1 et A2 de l'appendice).

\section{Résultats}

Vingt-quatre articles publiés avant le 25 janvier 2021 sur l'incidence de la fermeture ou de la réouverture des écoles sur la propagation de la COVID-19 ont été identifiés. II s'agissait notamment d'une étude transversale (16), de deux études de cohortes $(17,18)$, de deux études d'agrégats spatio-temporels et d'éclosions $(19,20)$ et de 19 études écologiques. Onze de ces études sont des prépublications ou des études qui n'ont pas encore fait l'objet d'un examen par les pairs. Toutes les études mentionnées dans le présent examen sont antérieures à la détermination des variants préoccupants.

La plupart des études d'observation évaluant l'incidence de la fermeture et de la réouverture des écoles sur la propagation de COVID-19 dans les écoles n'ont signalé aucun effet significatif (voir le tableau A1 de l'appendice). Quatre études n'ont révélé aucune différence dans l'incidence des cas avant et après la fermeture des écoles pour les vacances, après les enfants qui sont restés à la maison par rapport à ceux qui sont allés à l'école avec une surveillance stricte, ou après la réouverture de l'école (16-19). Une étude d'enquête sur les éclosions a révélé une forte éclosion d'une école secondaire en Israël, mais cela a été faussé par le fait que le mandat du masque a été levé tout comme il y a eu une vague de chaleur, ce qui a pu affecter le respect d'autres mesures de santé publique recommandées (20). En outre, il a été noté qu'il y avait une surpopulation au secondaire qui limitait la distanciation physique et que les activités parascolaires n'étaient pas interdites.

Parmi les études écologiques évaluant la transmission communautaire (voir le tableau A2 de l'appendice), dix ont été menées dans plusieurs pays, cinq aux États-Unis, deux en Asie et deux en Europe. Cinq études ont révélé que la fermeture et la réouverture des écoles n'étaient pas significativement associées à la réduction de la transmission et de l'incidence de COVID-19 et qu'elles étaient beaucoup moins efficaces pour réduire la transmission comparativement aux autres interventions non pharmaceutiques (21-25). Quatre études ont signalé une réduction de l'incidence de COVID-19 dans la communauté allant de $8 \%$ à $62 \%$ après la fermeture des écoles (26-29). $D^{\prime}$ autres études ont fait état d'une réduction significative du taux de reproduction net $\left(R_{t}\right)(30-32)$. Trois études ont attribué des réductions importantes de la mortalité à la fermeture des écoles $(29,33,34)$ et une étude a signalé une augmentation de la mortalité avec la fermeture retardée des écoles (35). 


\section{Discussion}

Dans l'ensemble, les données de ces études étaient mitigées et variées en raison de plusieurs facteurs. D'après les résultats des études d'observation évaluant l'incidence de la COVID-19 dans les écoles, la fermeture et la réouverture des écoles n'ont pas contribué de façon significative à la transmission de la COVID-19 lorsque des mesures de prévention et contrôle des infections (PCl) ont été mises en œuvre dans les écoles. Les mesures de $\mathrm{PCl}$ mises en œuvre par les écoles étaient similaires dans la plupart des études d'observation et comprenaient des masques, la distanciation physique, un nettoyage fréquent, des classes réduites et une meilleure hygiène des mains. La mise en œuvre de ces mesures dans les écoles a été considérée comme une variable médiatrice en raison de la réduction de la transmission et du risque d'infection par les mesures de $\mathrm{PCl}(36)$.

Les résultats des études écologiques évaluant la transmission dans la collectivité étaient incohérents, certaines études indiquant que la fermeture et la réouverture des écoles n'étaient pas significativement associées à la réduction de la transmission (21-25), et d'autres études signalant une réduction significative des $R_{t}(30-32)$ et de la mortalité $(29,33,34)$. Dans plusieurs de ces études écologiques, il a été signalé que d'autres interventions non pharmaceutiques comme le confinement, I'interdiction de rassemblements, l'obligation du port du masque, la fermeture des commerces non essentiels et les restrictions de voyage étaient plus efficaces que les fermetures d'écoles pour réduire la transmission de la COVID-19. Les études écologiques sont considérées comme un faible niveau de preuves en raison de la conception de la recherche, des facteurs de confusion multiples et du degré élevé de variabilité des résultats. Toutes les études écologiques incluses dans cet examen ont analysé les données sur la fermeture et la réouverture des écoles au début de la pandémie, entre janvier et août 2020, lorsque plusieurs interventions non pharmaceutiques ont été mises en œuvre simultanément. Par conséquent, il n'a pas été possible d'isoler l'incidence de la fermeture ou de la réouverture des écoles sur le nombre de cas de COVID-19 dans la collectivité. De plus, une seule des études écologiques a décrit le respect des mesures de $\mathrm{PCl}$ dans les écoles (25). Ces facteurs contribuent probablement à l'hétérogénéité entre les études.

Une des limites importantes de cet examen était les incohérences dans les niveaux d'enseignement qui ont été inclus dans chaque étude, ce qui a peut-être augmenté la variabilité des mesures de l'efficacité des fermetures d'écoles dans toutes les études. La plupart des études n'ont pas fourni d'information sur les écoles qui ont été incluses pour déterminer l'incidence de la fermeture des écoles sur la propagation de COVID-19. Certaines études mesuraient uniquement la fermeture des écoles primaires et secondaires et d'autres les mesuraient en combinaison avec les écoles postsecondaires. Le risque de transmission peut avoir varié considérablement entre les élèves des écoles primaires et secondaires en raison de différences potentielles dans leurs comportements et de l'adhésion aux mesures de $\mathrm{PCl}$ avec une différence résultante dans la réduction de la transmission virale. On a constaté que la transmission était plus faible dans les écoles primaires que dans les écoles secondaires, d'après les résultats d'une étude menée dans le cadre d'un examen qui l'a évaluée (19). Bien qu'ils ne soient pas expressément mentionnés dans l'étude précédente, leurs résultats concordaient avec ce que nous savons sur l'utilisation de $\mathrm{PCl}$ pour limiter la transmission dans ces paramètres (37). De plus, il a été démontré que l'impact relatif de la fermeture des écoles et de la réouverture varie selon le moment de la mise en œuvre, le niveau de transmission communautaire et la structure des populations de différents pays.

D'après les données empiriques résumées dans le présent article, les fermetures d'écoles ont eu un faible effet sur la limitation de la propagation de la COVID-19 dans les écoles et la communauté et semblent être beaucoup moins efficaces que les autres interventions non pharmaceutiques. Ces constatations sont également conformes aux études de modélisation menées partout au Canada (9-15). La mise en œuvre de la fermeture des écoles est actuellement fondée sur le moment où la transmission de COVID-19 dans la collectivité est élevée, comme le dictent les administrations sanitaires locales; toutefois, le rôle de la fermeture et de la réouverture des écoles dans les régions à faible transmission communautaire est moins clair et devrait davantage être étudié.

La fermeture des écoles peut être associée à des effets négatifs sur la santé mentale des élèves et le progrès scolaire (2); ainsi, les décideurs en santé publique devraient se demander si la faible efficacité apparente de la fermeture des écoles pour réduire la transmission l'emporte sur les nombreuses conséquences négatives sur le bien-être des élèves. Dans l'ensemble, la confiance dans ces données est faible, étant donné que les études présentées dans le présent examen varient selon plusieurs facteurs et ont été menées à différents moments et dans plusieurs pays. Enfin, la période d'étude de cet examen est également une limite, car il existe des différences marquées entre les $3^{\mathrm{e}}$ et $4^{\mathrm{e}}$ vagues par rapport aux $1^{\mathrm{re}}$ et $2^{\mathrm{e}}$ vagues de COVID-19 avec l'introduction de variants préoccupants plus transmissibles. La façon dont la présente preuve se comparera à celle obtenue pendant les périodes de propagation de variants préoccupants plus transmissibles n'est pas connue pour le moment et nécessitera une étude plus approfondie.

\section{Conclusion}

Les résultats de cet examen peuvent avoir des répercussions sur la prise de décisions en santé publique et sur les recherches futures sur les stratégies d'atténuation pour les écoles. Les données préliminaires fournies dans le cadre de cet examen laissent entendre que la fermeture et la réouverture des écoles peuvent avoir une incidence limitée sur la transmission de la COVID-19 au sein d'une collectivité. Cependant, il y a toujours un degré élevé d'incertitude en raison de la forte variation de 
la méthodologie et des résultats dans les diverses études. Des recherches supplémentaires sont nécessaires pour explorer plus systématiquement les répercussions de la fermeture des écoles et déterminer comment et quand elle peut être utilisée le plus efficacement pour lutter contre l'épidémie.

Les principales lacunes en matière de connaissances à considérer sont les suivantes : 1) la présence de nouveaux variants préoccupants et 2 ) le déploiement des vaccins contre la COVID-19 aura une incidence sur la transmission de la COVID-19 dans les écoles et les communautés. Les données présentées dans cet article précèdent l'introduction de variants préoccupants; par conséquent, des recherches supplémentaires sont nécessaires pour comprendre comment l'émergence et la propagation de ces variants auront une incidence sur l'efficacité de la fermeture des écoles ou sur l'incidence de la réouverture des écoles sur la propagation de COVID-19.

\section{Déclaration des auteurs}

M. E. J. - Méthodologie, enquête, rédaction de l'ébauche originale

R. E. - Conceptualisation, rédaction et révision, supervision

L. W. - Rédaction-examen et révision

P. H. - Rédaction-examen et révision

N. A. - Rédaction-examen et révision

M. S. - Rédaction-examen et révision

S. B.-A. - Rédaction-examen et révision

\section{Intérêts concurrents}

Aucun.

\section{Remerciements}

Nous tenons à souligner le travail du Groupe des sciences émergentes qui nous a permis de collaborer avec eux sur cette question importante.

\section{Financement}

Aucun.

\section{Références}

1. Organisation mondiale de la santé. Directeur général de I'OMS lors du point presse sur la COVID-19 - 11 mars 2020. Genève (CH) : OMS; 2020 (accédé 2021-05-27). https:// www.who.int/fr/director-general/speeches/detail/whodirector-general-s-opening-remarks-at-the-media-briefingon-covid-19---11-march-2020
2. Viner R, Russell S, Saulle R, Croker H, Stansfeld C, Packer J, Nicholls D, Goddings A-L, Bonell C, Hudson L, Hope S, Schwalbe N, Morgan A, Minozzi S. Impacts of school closures on physical and mental health of children and young people: a systematic review. medRxiv. $2021 ; 2021.02 .10 .21251526$. DOI

3. Bahn GH. Coronavirus disease 2019, school closures, and children's mental health. Soa Chongsonyon Chongsin Uihak 2020;31(2):74-9. DOI PubMed

4. Lee J. Mental health effects of school closures during COVID-19. Lancet Child Adolesc Health 2020;4(6):421. DOI PubMed

5. London School Economics and Polital Science. Eyles A, Gibbons S, Montebruno P. Covid-19 school shutdowns: What will they do to our children's education? LSE; 2020. http://eprints.Ise.ac.uk/104675/3/Eyles_covid_19_school_ shutdowns_published.pdf

6. Kuhfeld M, Soland J, Tarasawa B, Johnson A, Ruzek E, Liu J. Projecting the Potential Impact of COVID-19 School Closures on Academic Achievement. Educ Res 2020;49(8):549-65. DOl

7. Centre de collaboration nationale des maladies infectieuses. Isfeld-Kiely H, Moghadas S. Efficacité de la fermeture des écoles comme mesure de lutte contre la grippe: Analyse des données probantes récentes. CCNMI; 2014.

https://ccnmi.ca/publications/efficacite-de-la-fermeture-desecoles-comme-mesure-de-lutte-contre-la-grippe/

8. World Health Organization. Non-pharmaceutical public health measures for mitigating the risk and impact of epidemic and pandemic influenza. Geneva (CH): WHO; 2019 (accédé 2021-04-27). http://www.who.int/influenza/ publications/public_health_measures/publication/en/

9. Abdollahi E, Haworth-Brockman M, Keynan Y, Langley JM, Moghadas SM. Simulating the effect of school closure during COVID-19 outbreaks in Ontario, Canada. BMC Med 2020;18(1):230. DOI PubMed

10. Naimark D, Mishra S, Barrett K, Khan YA, Mac S, Ximenes R, Sander B. Simulation-Based Estimation of SARS-CoV-2 Infections Associated With School Closures and Community-Based Nonpharmaceutical Interventions in Ontario, Canada. JAMA Netw Open 2021;4(3):e213793. DOI PubMed

11. Ng V, Fazil A, Waddell LA, Bancej C, Turgeon $P_{,}$ Otten A, Atchessi N, Ogden NH. Projected effects of nonpharmaceutical public health interventions to prevent resurgence of SARS-CoV-2 transmission in Canada. CMAJ 2020;192(37):E1053-64. DOI PubMed

12. Davies NG, Kucharski AJ, Eggo RM, Gimma A Edmunds WJ; Centre for the Mathematical Modelling of Infectious Diseases COVID-19 working group. Effects of non-pharmaceutical interventions on COVID-19 cases, deaths, and demand for hospital services in the UK: a modelling study. Lancet Public Health 2020;5(7):e375-85. DOI PubMed 
13. Koo JR, Cook AR, Park M, Sun Y, Sun H, Lim JT, Tam C, Dickens BL. Interventions to mitigate early spread of SARS-CoV-2 in Singapore: a modelling study. Lancet Infect Dis 2020;20(6):678-88. DOI PubMed

14. Courtemanche C, Garuccio J, Le A, Pinkston J, Yelowitz A. Strong social distancing measures in the United States reduced the covid-19 growth rate. Health Aff (Millwood) 2020;39(7):1237-46. DOI PubMed

15. Garchitorena A, Gruson H, Cazelles B, Karki T, Sudre B, Roche B. Integrated packages of non-pharmaceutical interventions increased public health response efficiency against COVID-19 during the first European wave: evidence from 32 European countries. medRxiv. 2020; 2020.08.17.20174821v2. DOI

16. Kriger O, Lustig Y, Cohen C, Amit S, Biber A, Barkai G, Talmi L, Gefen-Halevi S, Mechnik B, Regev-Yochay G. The Sheba Medical Center healthcare workers' children's school: can we open schools safely? Clin Microbiol Infect 2021;27(3):474.e1-3. DOI PubMed

17. Gandini S, Rainisio M, Luisa lannuzzo M, Bellerba F, Cecconi F, Scorrano L. No evidence of association between schools and SARS-CoV-2 second wave in Italy. med Rxiv. 2020; 2020.12.16.20248134v2. DOI

18. Fontanet A, Grant R, Tondeur L, Madec Y, Grzelak L, Cailleau I, Ungeheuer M-N, Renaudat C, Fernandes Pellerin S, Kuhmel L, Staropoli I, Anna F, Charneau P, Demeret C, Bruel T, Schwartz O, Hoen B. SARS-CoV-2 infection in primary schools in northern France: A retrospective cohort study in an area of high transmission. medRxiv. 2020;2020.06.25.20140178v2. DOI

19. Larosa E, Djuric O, Cassinadri M, Cilloni S, Bisaccia E, Vicentini M, Venturelli F, Giorgi Rossi P, Pezzotti P, Bedeschi E; Reggio Emilia Covid-19 Working Group. Secondary transmission of COVID-19 in preschool and school settings in northern Italy after their reopening in September 2020: a population-based study. Euro Surveill 2020;25(49):2001911. DOI PubMed

20. Stein-Zamir C, Abramson N, Shoob H, Libal E, Bitan M, Cardash T, Cayam R, Miskin I. A large COVID-19 outbreak in a high school 10 days after schools' reopening, Israel, May 2020. Euro Surveill 2020;25(29):2001352. DOI PubMed

21. An B, Porcher S, Tang SY, Kim E. Effects of Early Mask Mandates and Other Policy Interventions on COVID-19 Infections. Research Square. 2021. DOI

22. Liu X, Xu X, Li G, Xu X, Sun Y, Wang F, Shi X, Li X, Xie G, Zhang L. Differential impact of non-pharmaceutical public health interventions on COVID-19 epidemics in the United States. BMC Public Health 2021;21(1):965. DOI PubMed

23. Iwata K, Doi A, Miyakoshi C. Was school closure effective in mitigating coronavirus disease 2019 (COVID-19)? Time series analysis using Bayesian inference. Int $\mathrm{J}$ Infect Dis 2020;99:57-61. DOI PubMed

24. Wieland T. A phenomenological approach to assessing the effectiveness of COVID-19 related nonpharmaceutical interventions in Germany. Saf Sci 2020;131:104924. DOI PubMed
25. Ehrhardt J, Ekinci A, Krehl H, Meincke M, Finci I, Klein J, Geisel B, Wagner-Wiening C, Eichner M, Brockmann SO. Transmission of SARS-CoV-2 in children aged 0 to 19 years in childcare facilities and schools after their reopening in May 2020, Baden-Württemberg, Germany. Euro Surveill 2020;25(36):2001587. DOI PubMed

26. Banholzer N, van Weenen $E$, Lison A, Cenedese A, Seeliger A, Kratzwald B, Tschernutter D, Salles JP, Bottrighi P, Lehtinen S, Feuerriegel S, Vach W. Estimating the effects of non-pharmaceutical interventions on the number of new infections with COVID-19 during the first epidemic wave. PLoS One 2021;16(6):e0252827. DOI PubMed

27. Banholzer N, van Weenen E, Kratzwald B, Seeliger A, Tschernutter D, Bottrighi P, Cenedese A, Salles JP, Vach W, Feuerriegel S. Impact of non-pharmaceutical interventions on documented cases of COVID-19. medRxiv. 2020; 2020.04.16.20062141v3. DOI

28. Brauner JM, Mindermann S, Sharma M, Johnston D, Salvatier J, Gavenčiak T, Stephenson AB, Leech G, Altman G, Mikulik V, Norman AJ, Monrad JT, Besiroglu T, Ge H, Hartwick MA, Teh YW, Chindelevitch L, Gal Y, Kulveit J. Inferring the effectiveness of government interventions against COVID-19. Science 2021;371(6531):eabd9338. DOl PubMed

29. Auger KA, Shah SS, Richardson T, Hartley D, Hall M, Warniment A, Timmons K, Bosse D, Ferris SA, Brady PW, Schondelmeyer AC, Thomson JE. Association Between Statewide School Closure and COVID-19 Incidence and Mortality in the US. JAMA 2020;324(9):859-70. DOI PubMed

30. Esra R, Jamieson L, Fox MP, Letswalo D, Ngcobo N, Mngadi S, Estill J, Meyer-Rath G, Keiser O. Evaluating the impact of non-pharmaceutical interventions for SARS-CoV-2 on a global scale. medRxiv 2020; 2020.07.30.2016439. DOI

31. Dreher N, Spiera Z, Mcauley FM, Kuohn L, Durbin JR, Marayati NF, Ali M, Li AY, Hannah TC, Gometz A, Kostman JT, Choudhri TF. Impact of policy interventions and social distancing on SARS-CoV-2 transmission in the United States. medRxiv. 2020; 2020.05.01.20088179v1. DOI

32. Cowling BJ, Ali ST, Ng TW, Tsang TK, Li JC, Fong MW, Liao Q, Kwan MY, Lee SL, Chiu SS, Wu JT, Wu P, Leung GM. Impact assessment of non-pharmaceutical interventions against coronavirus disease 2019 and influenza in Hong Kong: an observational study. Lancet Public Health 2020;5(5):e279-88. DOI PubMed

33. Stokes, J, Turner AJ, Anselmi L, Morciano M, Hone T. The relative effects of non-pharmaceutical interventions on early Covid-19 mortality: natural experiment in 130 countries. medRxiv.2020; 2020.10.05.20206888v1. DOI

34. Pasdar Z, Pana TA, Ewers KD, Szlachetka WA, Perdomo-Lampignano JA, Gamble DT, Bhattacharya S, Carter B, Myint PK. An Ecological Study Assessing the Relationship between Public Health Policies and Severity of the COVID-19 Pandemic. SSRN Electron J. 2020. https://papers.ssrn.com/abstract $=3634847$ 
35. Yehya N, Venkataramani A, Harhay MO. Statewide Interventions and Coronavirus Disease 2019 Mortality in the United States: An Observational Study. Clin Infect Dis 2021;73(7):e1863-9. DOl PubMed

36. Santé publique Ontario. Recommandations en $\mathrm{PCl}$ concernant l'utilisation d'équipements de protection individuelle pour la prise en charge des personnes dont I'infection à la COVID-19 est suspectée ou confirmée. Toronto (ON) : SPO; 2021. https://www.publichealthontario. $\mathrm{ca} /$-/media/documents/ncov/updated-ipac-measurescovid-19.pdf?la=fr

37. Centre de collaboration nationale des méthodes et outils. Revue rapide évolutive, mise à jour 17: Quel rôle particulier jouent les garderies et les écoles dans la transmission de la COVID-19? Hamilton (ON) : CCNMO; 2021 (accédé 2021-10-28). https://www.nccmt.ca/fr/covid-19/covid-19rapid-evidence-service/19
38. Klimek-Tulwin M, Tulwin T. Early school closures can reduce the first-wave of the COVID-19 pandemic development. J Public Health (Berl.) 2020. DOI

39. Papadopoulos DI, Donkov I, Charitopoulos K, Bishara S. The impact of lockdown measures on COVID-19: a worldwide comparison. medRxiv. 2020; 2020.05.22.20106476v2. DOI

40. Jüni $P$, Rothenbühler $M$, Bobos $P$, Thorpe $K E$, da Costa $B R$, Fisman DN, Slutsky AS, Gesink D. Impact of climate and public health interventions on the COVID-19 pandemic: a prospective cohort study. CMAJ 2020;192(21):E566-73. DOI PubMed

41. Krishnamachari B, Morris A, Zastrow D, Dsida A, Harper B, Santella AJ. Effects of Government Mandated Social Distancing Measures on Cumulative Incidence of COVID-19 in the United States and its Most Populated Cities. medRxiv. 2020; 2020.05.22.20110460v1. DOI 


\section{Appendice : Tableaux}

\section{Tableau A1 de l'appendice : Résumé des études d'observation évaluant l'incidence de la fermeture ou de la réouverture des écoles sur la transmission de COVID-19 dans les écoles et la communauté $(n=5)$}

\section{Étude}

Méthode
Principaux résultats

Études de cohortes $(n=2)$

Gandini (2020) (17)

Étude de cohorte prospective et étude transversale

Italie

Sept.-nov. 2020

Étude rétrospective de cohorte

France

Fév.-avr. 2020
Cette étude a analysé l'association entre les dates de réouverture des écoles et les cas de COVID-19 dans vingt et une régions italiennes à l'aide d'une base de données sur les cas positifs dans les écoles primaires, intermédiaires et secondaires et l'incidence du SRAS-CoV-2 dans la population générale. Les mesures de $\mathrm{PCl}$ comprenaient le contrôle de la température, I'hygiène des mains, le port obligatoire du masque pour les élèves et le personnel, la distanciation physique, l'interdiction des sports et de la musique et la réduction de la durée de l'école.

Plusieurs résultats de COVID-19 ont été mesurés lors de la réouverture des écoles : croissance de l'incidence, le $\mathrm{R}_{\mathrm{t}^{\prime}}$ et les infections secondaires

Cette étude de cohorte rétrospective comprenait des élèves de l'école primaire, des enseignants, du personnel non enseignant, des parents et des parents exposés au SRAS-CoV-2 en février et en mars dans six écoles. Les mesures de $\mathrm{PCl}$ n'ont pas été décrites.

Les participants ont rempli un questionnaire portant sur l'information sociodémographique et les antécédents de symptômes récents. Des échantillons de sang ont également été testés pour détecter la présence d'anticorps anti-SRAS-CoV-2 au moyen d'un essai fondé sur la cytométrie en flux. Trois introduction du SRAS-CoV-2 ont eu lieu avant la fermeture des écoles. Cette étude séroépidémiologique a porté sur la propagation dans les écoles par rapport aux familles. On a comparé le TAl entre les contacts scolaires et les contacts familiaux afin de comprendre l'incidence possible de la fermeture de l'école.
Rien n'indique que la deuxième vague de SRAS-CoV-2 ait été provoquée par la réouverture des écoles dans les régions.

L'incidence du SRAS-CoV-2 chez les étudiants était inférieure à la population générale de toutes les régions italiennes, sauf deux.

L'augmentation du $R_{t}$ n'était pas associée aux différentes dates d'ouverture des écoles.

La fermeture des écoles mises en œuvre dans deux régions n'a pas eu d'incidence sur le déclin du $R_{t}$.

e TAl était de 45/510 (8,8\%),3/42 (7,1\%), 1/28 (3,6\%) $76 / 641(11,9 \%)$ et $14 / 119(11,8 \%)$ chez les élèves de l'école primaire, les enseignants, le personnel non enseignant, les parents et les membres de la famille $(p=0,29)$.

Aucune infection secondaire due à l'introduction de COVID-19 dans les écoles n'a été détectée chez les élèves et les enseignants.

Parmi les élèves infectés, leurs parents étaient beaucoup plus susceptibles d'être infectés $(61,0 \%$ contre $6,9 \% ; p<0,0001)$ la même chose a été observée chez les parents d'élèves infectés par rapport aux élèves non infectés $(44,4 \%$ contre 9,1\%; $p=0,002$ )

La fermeture des écoles ne semble pas avoir eu d'incidence sur la transmission.

Études transversales $(n=1)$

Kriger (2020) (16)

Étude transversale

Israël

Mars-mai 2020
Lors d'un confinement national, une école alternative a été utilisée pour que les enfants des travailleurs de la santé puissent suivre une surveillance stricte des symptômes. Les familles avec enfants restées à la maison ont été comparées aux enfants de cette école alternative. Les mesures de $\mathrm{PCl}$ à l'école comprenaient la désinfection quotidienne, l'utilisation de masque facial par le personnel et le lavage fréquent des mains.

Cette étude transversale comprenait 70 enfants qui ont fréquente l'école primaire alternative et 36 qui sont restés à la maison, ainsi que leurs 78 parents.

Les données ont été recueillies au moyen d'un court questionnaire; des écouvillons nasopharyngés et oropharyngés ont été obtenus et testés pour le SRAS-CoV-2 par RT-PCR, et du sang a été recueilli pour les titres d'lgA et d'lgG du SRAS-CoV-2.

\section{Enquêtes sur les agrégats spatio-temporels et les éclosions $(n=2)$}

Larosa (2020) (19) Cette enquête en grappe a analysé la transmission de COVID-19

Enquête sur les agrégats spatio-temporels

Italie

Sept.-oct. 2020

Stein-Zamir (2020)

(20)

Enquête sur l'éclosion Israël

Mai-juin 2020 élèves de la $7^{\mathrm{e}}$ à $12^{\mathrm{e}}$ années. dans 41 classes de 36 écoles lors de leur réouverture dans le nord de l'Italie. Le taux d'attaque secondaire a été mesuré chez les élèves et les enseignants des écoles primaires et secondaires (écoles intermédiaires et secondaires). Les mesures du PCl comprenaient : le port du masque obligatoire pour les élèves du secondaire seulement, la distanciation physique et l'interdiction des activités parascolaires.

Cette étude d'enquête sur l'éclosion a évalué les caractéristiques épidémiologiques d'une éclosion au secondaire à Jérusalem qui a montré une transmission massive de COVID-19 lors de la réouverture de l'école le 17 mai. L'école secondaire comprenait des

Une vague de chaleur extrême s'est produite lors de la réouverture de l'école. Mesures de $\mathrm{PCl}$ : I'utilisation du masque a été levée pendant trois jours pendant la vague de chaleur, la distanciation physique était en dessous des normes dans les classes surpeuplées et les activités parascolaires n'ont pas été interdites.
Des symptômes ont été signalés chez environ $16 \%$ des enfants des deux groupes : ceux qui ont fréquenté l'école ( $n=11 / 70$ ) et ceux qui n'y ont pas fréquenté $(n=6 / 36)$.

Des tests sérologiques positifs montrant une exposition antérieure ont été détectés dans moins de $2 \%$ de chaque groupe et ils n'étaient pas significativement différents les uns des autres.

Il n'y avait aucune preuve d'une augmentation de l'infection chez les élèves par rapport à ceux à la maison.

Le taux d'attaques secondaires causées par la COVID-19 a été plus élevé dans les écoles secondaires $(6,6 \%)$ que dans les écoles primaires $(0,38 \%)$

Il a été signalé que la proportion des 10 à 19 ans était de $19,8 \%(n=938 / 4747)$ des cas avant le 24 mai, puis augmentait à $40,9 \%(n=316 / 772)$ après le 24 mai.

Les tests de l'ensemble de l'école ont révélé que 153 élèves (taux d'attaque : 13,2\%) et 25 membres du personnel (taux d'attaque : $16,6 \%$ ) ont été positifs à la COVID-19. Les taux de COVID-19 étaient plus élevés chez les élèves de la $7^{\mathrm{e}}$ à la $9^{\mathrm{e}}$ année que ceux de la $10^{\mathrm{e}}$ à la $12^{\mathrm{e}}$ année. 


\section{Tableau A2 de l'appendice : Résumé des études écologiques évaluant l'efficacité des fermetures d'écoles ou des réouvertures pour réduire la propagation de la COVID-19 dans la collectivité $(n=19)$}

\begin{tabular}{|c|c|}
\hline Étude & Méthode \\
\hline \multicolumn{2}{|l|}{ Global $(n=10)$} \\
\hline $\begin{array}{l}\text { An (2021) (21) } \\
\text { Étude écologique } \\
\text { Mondial } \\
\text { Janv.-juill. } 2020\end{array}$ & $\begin{array}{l}\text { Cette étude visait à identifier les associations entre six INP et le nombre } \\
\text { d'infections à la COVID-19. À l'aide de données mondiales sur les INP et } \\
\text { les infections à la COVID-19 entre janvier et juillet 2020, une analyse a été } \\
\text { menée sur les effets à court et à long terme des INP sur les nouveaux taux } \\
\text { d'infection 5, 9, } 12 \text { et } 21 \text { jours après leur adoption. Les mesures de PCl et le } \\
\text { niveau de scolarité inclus dans l'étude n'ont pas été décrits. } \\
\text { Les INP examinées comprenaient le port obligatoire du masque, } \\
\text { des restrictions de voyage internationales, un confinement national, } \\
\text { l'interdiction de rassemblements, la fermeture des restaurants et la } \\
\text { fermeture des écoles. }\end{array}$ \\
\hline $\begin{array}{l}\text { Banholzer }(2020)(27) \\
\text { Étude écologique } \\
20 \text { pays }\end{array}$ & $\begin{array}{l}\text { Dans la présente étude, l'incidence des INP sur la réduction relative des } \\
\text { nouveaux cas de COVID-19 à l'aide d'un modèle bayésien hiérarchique } \\
\text { avec un effet temporel pour chaque INP. Les mesures de } \mathrm{PCI} \text { n'ont pas été } \\
\text { décrites. }\end{array}$ \\
\hline Avr. 2020 & $\begin{array}{l}\text { Les INP examinées comprenaient 1) la fermeture des écoles primaires, } \\
\text { 2) la fermeture des frontières, 3) l'interdiction d'événements publics, } \\
\text { 4) l'interdiction de rassemblement, 5) la fermeture de lieux, 6) l'interdiction } \\
\text { d'allées et venues publiques sans raison valable et 7) l'interdiction de travail } \\
\text { des activités commerciales non essentielles. }\end{array}$ \\
\hline
\end{tabular}

Banholzer (2021) (26) À l'aide d'un modèle bayésien hiérarchique semi-mécaniste, cette étude

Étude écologique

20 pays

Fév.-mai 2020 visait à mesurer l'efficacité de sept INP dans la réduction du nombre de nouvelles infections. Les mesures de $\mathrm{PCl}$ n'ont pas été décrites.

Les INP examinées comprenaient 1) la fermeture des écoles primaires, 2) la fermeture des frontières, 3) l'interdiction d'événements publics, 4) I'interdiction de rassemblement, 5) la fermeture de lieux, 6) l'interdiction d'allées et venues publiques sans raison valable et 7) l'interdiction de travail des activités commerciales non essentielles.

Brauner (2021) (28) Cette étude a estimé l'efficacité des INP dans 41 pays à l'aide d'un modèle Étude écologique 41 pays Janv.-mai 2020 bayésien hiérarchique en liant les dates de mise en œuvre de l'intervention au nombre national de cas et de décès.

Les tailles des effets d'intervention ont été catégorisées par les réductions médianes du $\mathrm{R}_{t}$ de moins de $17,5 \%$ (petite), entre $17,5 \%$ et $35 \%$ (modérée) et au moins $35 \%$ (grande). Les INP examinées comprenaient : 100 personnes ou moins, fermer certains commerces, fermer la plupart des commerces, fermer des écoles et des universités et rester à la maison. Les mesures de $\mathrm{PCl}$ n'ont pas été décrites.

Klimek-Tulwin (2020) (38) Cette étude visait à évaluer l'effet de la fermeture des écoles sur les cas de COVID-19 à l'échelle mondiale en mesurant la corrélation entre le taux d'incidence le jour de la fermeture de l'école et le taux d'incidence dans les jours suivants. Les mesures de $\mathrm{PCl}$ et le niveau de scolarité inclus dans l'étude n'ont pas été décrits. limiter les rassemblements à moins de 1000 personnes ou moins de

La fermeture des écoles a démontré une efficacité plus tardivement que d'autre INP. Après un certain temps, l'incidence de la fermeture des écoles sur les nouveaux taux de cas était de $-0,492(E T=0,16)$ à 12 jours $(p<0,01)$, de $-0,722(E T=0,148)$ à 21 jours $(p<0,001)$ et de $-0,824(E T=0,0967)$ à 30 jours $(p<0,001)$.

La fermeture des écoles n'a pas eu d'effets importants sur les infections ajustées en fonction de la population à long terme $\left(90^{\mathrm{e}}\right.$ à $120^{\mathrm{e}}$ jour $)$.

La réduction moyenne des nouveaux cas de COVID-19 avec fermeture d'écoles primaires était de $8 \%$ (IC à $95 \%$ : $0 \%-23 \%$ ).

Comparativement aux autres INP examinées, les fermetures d'écoles semblaient être l'un des INP les moins efficaces.

La réduction relative des nouveaux cas de COVID-19 avec la fermeture des écoles primaires était de $17 \%$ (IC à $95 \%$ : $2 \%-36 \%$ ).

Cette réduction était inférieure à deux autres INP (interdiction d'événements et fermeture de lieux).

La réduction en pourcentage du $R_{t}$ associée à la fermeture des écoles et des universités conjointement était de $38 \%$ (IC à $95 \%$ : 16\%-54\%), qui a été classé comme un effet de grande taille.

Les effets individuels des fermetures d'écoles n'ont pas été mesurés.

Les résultats indiquent qu'il y a une forte corrélation entre le jour de fermeture des établissements d'enseignement et le taux d'incidence dans les jours suivants $\left(16^{\mathrm{e}}[p=0,004], 30^{\mathrm{e}}[p=0,002]\right.$ et $60^{\circ}$ $[p=0,031]$ jours depuis le $100^{\mathrm{e}}$ cas confirmé dans chaque pays).

La fermeture précoce des écoles est statistiquement significativement corrélée avec des taux d'incidence plus faibles au cours des différentes phases de l'épidémie.

Fermeture des premières écoles généralisées $(p=0,050$, coefficient de régression $B=-0.012, I C$ à $95 \%: 0 \%-0,024 \%)$ était associé à une réduction du Lincidence des mesures de confinement a été évaluée à l'échelle mon à l'aide de données accessibles au public. On a comparé le moment et I'association des premiers INP avec $\log _{10}$ les décès $\left(\log _{D}\right)$ et $\log _{10}$ les cas (2020) (39) nationaux ( $\log _{C}$ ) entre les pays. Les mesures de $\mathrm{PCl}$ et le niveau de scolarité Étude écologique Mondial inclus dans l'étude n'ont pas été décrits.

Janv.-avr. 2020

Pasdar (2020) (34) Étude écologique 22 pays Mai 2020
L'objectif de cette étude était de déterminer les liens entre les INP et les résultats de COVID-19.

Les associations avec des INP ont été évaluées avec leur indice de rigueur respectif sur plusieurs résultats qui forment la courbe de l'épidémie : taux de mortalité moyen, temps jusqu'au pic, décès au pic par 100000 habitants, nombre cumulatif de décès après pic par 100000 habitants et ratio de la pente moyenne de la courbe descendante à la pente moyenne de la courbe ascendante. Les mesures de $\mathrm{PCl}$ et le niveau de scolarité inclus dans l'étude n'ont pas été décrits.
La fermeture des écoles était efficace contre tous les résultats, sauf le temps nécessaire pour atteindre le pic de la courbe de l'épidémie.

La plus forte association a été observée dans les décès cumulatifs après le pic, pour 100000 ( $r s=-0,744, p=0,009$ ).

Dans les pays non européens, les fermetures d'écoles ont été les plus efficaces par rapport au taux de mortalité moyen ( $r s=-0,757, p=0,049$ ). 


\section{Tableau A2 de l'appendice : Résumé des études écologiques évaluant l'efficacité des fermetures d'écoles ou des réouvertures pour réduire la propagation de la COVID-19 dans la collectivité $(n=19)$ (suite)}

Étude

Méthode
Principaux résultats

Global $(n=10)$ (suite)

Esra (2020) (30)

Étude écologique

Mondial

Janv.-mai 2020

Cette étude a utilisé des données rapportées à l'échelle mondiale sur les cas de SRAS-CoV-2 pour s'adapter à un cadre de modèle bayésien afin d'estimer l'association avec les INP et la transmission.

Les INP examinées comprennent les mandats de séjour à domicile, les limites de collecte, la fermeture des écoles (établissements d'enseignement primaire, secondaire et supérieur) et les politiques de masquage. Les mesures de $\mathrm{PCl} n$ 'ont pas été décrites.

Jüni (2020) (40)

Étude écologique

Cette étude prospective des zones géopolitiques visait à déterminer si les interventions climatiques ou de santé publique sont associées à la réduction de la transmission de COVID-19.

Mondial

Mars 2020

Une régression pondérée des effets aléatoires a été utilisée pour déterminer l'association entre la croissance épidémique RT et les mesures climatiques et les interventions de santé publique comme la fermeture des écoles, les restrictions aux rassemblements de masse et les mesures de distanciation sociale au cours d'une période d'exposition 14 jours auparavant. Les mesures de $\mathrm{PCl}$ et le niveau de scolarité inclus dans l'étude n'ont pas été décrits.

Stokes (2020) (33)

Étude écologique

Cette étude a examiné la variation des INP dans 130 pays au cours de deux périodes : 1) avant le premier décès causé par la COVID-19 et

2) 14 jours après le premier décès causé par la COVID-19.

Mondial

La présente étude a examiné les associations avec des décès quotidiens causés par la COVID-19 par million et par période de 24 jours (période entre la transmission du virus et la mortalité). Les mesures de $\mathrm{PCl}$ et le niveau de scolarité inclus dans l'étude n'ont pas été décrits.

Amérique du Nord $(n=5)$

Auger (2020) (29)

Étude écologique

É.-U.

Mars-mai 2020

Dreher (2020) (31)

Étude écologique

É.-U.

Avr. 2020

Krishnamachari

(2020) (41)

Étude écologique

É.-U.

Mai 2020
Cette étude visait à déterminer si la fermeture des écoles était associée à une diminution de I'incidence cumulative de COVID-19 et de mortalité

L'incidence de la fermeture des écoles primaires et secondaires a été évaluée à l'aide de données accessibles au public provenant des 50 États. Les mesures de $\mathrm{PCl}$ n'ont pas été décrites.

Cette étude visait à mesurer l'incidence des INP sur le taux effectif de COVID-19 dans les États américains.

Le $R_{t}$ moyen a été mesuré au cours des semaines qui ont suivi l'atteinte de 500 cas par État. Le $R_{t}$ a été mesuré à la semaine suivant immédiatement le $500^{\circ}$ cas (jours +1 à +7 ) et à un délai d'une semaine à partir du $500^{\mathrm{e}}$ cas (jours +8 à +14 ).

Les INP examinées comprenaient le séjour à la maison, la fermeture d'établissements d'enseignement et la fermeture des commerces non essentiels. Les mesures de $\mathrm{PCl}$ et le niveau de scolarité inclus dans l'étude n'ont pas été décrits.

Cette étude visait à examiner les effets des IPN sur les taux cumulatifs d'incidence de COVID-19 aux États-Unis au niveau de l'État dans les 25 villes les plus peuplées, tout en tenant compte des facteurs de risque sociodémographiques.

Une régression binomiale négative a été utilisée pour calculer les ratios de taux ajustés en comparant deux niveaux d'une variable binaire : " au-dessus de la valeur médiane " et " à la valeur médiane et au-dessous » pour les jours précédant la mise en œuvre d'un INP. Les INP évalués dans cette étude comprenaient : jours avant la fermeture de commerces non essentiels, jours avant le confinement à domicile, jours après la fermeture des rassemblements, jours après la fermeture des restaurants et jours après la fermeture des écoles. Les mesures de $\mathrm{PCl}$ et le niveau de scolarité inclus dans l'étude n'ont pas été décrits.
II y a eu une réduction moyenne estimée de $\mathrm{R}$ de $12 \%$ (IC à $95 \%$ : $5 \%-19 \%$ ) avec la fermeture des écoles (établissements d'enseignement primaire, secondaire et tertiaire).

De fortes associations négatives avec une croissance épidémique ont été observées pour la fermeture des écoles (RT -0,63, IC à $95 \%$ : 0,52 \%-0,78\%)

Cette association était plus prononcée dans les domaines où deux ou trois INP ont été mis en œuvre que dans un seul INP.

La fermeture des écoles plus sévères ou antérieures était associée aux réductions les plus importantes de décès causé par la COVID-19 (-1,23 par million [IC à $95 \%$ : $-2,20 \%-0,27 \%]$ ) comparativement aux autres INP.
Les résultats ont montré que la fermeture des écoles était associée à une baisse importante de l'incidence de la COVID-19 (-62 \% [IC à $95 \%$ : $-71 \%-49 \%]$ ) et dans la mortalité (-58 \% [IC à $95 \%$ : -68 \%-46 \%])

Ces associations étaient plus fortes dans les États où I'incidence cumulative de COVID-19 était faible au moment de la fermeture de l'école.

La fermeture des établissements d'enseignement a été associée à une réduction significative de $R_{t}$ par rapport aux États qui n'avaient pas cette politique la semaine suivant 500 cas $(B=-0,17, \mathrm{IC}$ à $95 \%:-0,30 \%--0,05 \%$, $p=0,009)$

De 8 à 14 jours après la date du $500^{e}$ cas, la fermeture des établissements $d$ 'enseignement était associée à une réduction importante du $\mathrm{R}_{\mathrm{t}}$ comparativement aux contrôles ( $(=-0,12, I C$ à $95 \%$ : $-0,21 \%--0,04 \%$, $p=0,006)$

La fermeture des écoles a été associée à une incidence cumulative sur les jours 35 et 42 , avec un ratio de taux ajusté de 1,59 (IC à $95 \%$ : $1,03 \%-2,44 \%, p=0,04)$ à 35 jours, et ratio de taux rajusté de 1,64 (IC à $95 \%: 1,07 \%-2,52 \%, p=0,04$ ) à 42 jours.

Les retards dans la fermeture des écoles étaient positivement associés à l'incidence cumulative au niveau de l'État. 


\section{Tableau A2 de l'appendice : Résumé des études écologiques évaluant l'efficacité des fermetures d'écoles ou des réouvertures pour réduire la propagation de la COVID-19 dans la collectivité $(n=19)$ (suite)}

\section{Étude}

Méthode

Principaux résultats

Amérique du Nord ( $n=5$ ) (suite)

Liu (2020) (22)
Étude écologique
É.-U.

Cette étude a estimé l'impact de neuf INP différents sur la réduction du $\mathrm{R}_{\mathrm{t}}$ efficace en utilisant le nombre quotidien de nouveaux cas signalés et d'infections inférées dans 50 États. Les mesures de $\mathrm{PCl}$ et le niveau de scolarité inclus dans l'étude n'ont pas été décrits.
Fév.-avr. 2020

Yehya (2020) (35)

Étude écologique É.-U.

Janv.-avr. 2020
Dans cette étude, une analyse au niveau de l'état a été effectuée pour déterminer l'association entre les INP mis en œuvre ultérieurement et les taux de mortalité plus élevés.

À l'aide d'une régression binomiale négative multivariable, I'association a été testée entre le moment des déclarations d'urgence et la fermeture d'écoles avec une mortalité de 28 jours. Le jour 1 de chaque état était fixé à 10 morts ou plus. Les mesures de $\mathrm{PCl}$ et le niveau de scolarité inclus dans l'étude n'ont pas été décrits.
On a constaté que la fermeture des écoles réduisait modérément le $\mathrm{R}$ d'environ $10 \%$ (IC à $95 \%$ : $7 \%-14 \%)$.

Cette réduction était inférieure à six autres INP évalués (confinement à domicile obligatoire, port du masque, interdiction de rassemblement, fermeture de commerces non essentiels, déclaration d'état d'urgence et restriction de voyage entre États).

Plus tard, la fermeture des écoles était associée à un plus grand nombre de décès (taux de mortalité ajusté de 1,05; IC à $95 \%: 1,01 \%-1,09 \%$; $p=0,008)$.

\section{Asie $(n=2)$}

Cowling (2020) (32) Cette étude a examiné l'effet des interventions en santé publique sur Étude écologique Hong Kong Janv.-fév. 2020 I'incidence de COVID-19 et sur l'efficacité quotidienne du $R_{t}$ Les cas de COVID-19 confirmés en laboratoire et le $R_{t}$ quotidien effectif ont été estimés afin de déterminer les changements dans la transmissibilité au fil du temps. La fermeture des écoles comprenait des jardins d'enfants allant jusqu'aux établissements d'enseignement supérieur et postdoctoral, ainsi que des centres de tutorat. Les mesures de $\mathrm{PCl}$ n'ont pas été décrites.
La valeur estimative du $R_{t}$ était de 1,28 (IC à

$95 \%: 1,26 \%-1,30 \%$ ) au cours de la période de deux semaines précédant la fermeture des écoles et $0,72$ (IC à $95 \%: 0,70 \%-0,74 \%)$ au cours des deux premières semaines de la fermeture des écoles, ce qui correspond à un IC de $44 \%$ (95\% : $34 \%-53 \%)$ réduction de la transmissibilité.

Le $R_{t}$ calculé à partir des données sur I'hospitalisation était de 1,10 $(1,06-1,12)$ avant le début de la fermeture des écoles et réduit à 0,73 $(0,68-0,77)$ après la fermeture des écoles, ce qui correspond à un IC de $33 \%$ (95\% : $24 \%-43 \%)$ réduction de la transmissibilité.

L'intervention de fermeture des écoles n'a pas permis de réduire l'incidence de la COVID-19.

Les nouveaux cas de COVID-19 ont continué d'augmenter ( $\alpha-0,08$, IC à $95 \%$ : $-0,36 \%-0,65 \%)$.

\section{Japon}

Étude écolos

Mars 2020
Cette étude visait à évaluer l'efficacité de la fermeture des écoles primaires et secondaires sur l'incidence de la COVID-19 neuf jours après la mise en œuvre. Les mesures de $\mathrm{PCl}$ n'ont pas été décrites.

À l'aide d'une méthode bayésienne, des analyses de séries chronologiques ont été effectuées et des modèles de tendance linéaire locaux ont été élaborés pour le nombre de cas nouvellement signalés de COVID-19.

\section{Europe $(n=2)$}

Wieland (2020) (24) L'objectif de cette étude était d'évaluer l'efficacité des différents Étude écologique INP par rapport à la propagation de COVID-19 au fil du temps.

\section{Allemagne}

Mars-avr. 2020

La fermeture des écoles comprenait également la fermeture des garderies. Les mesures de $\mathrm{PCl}$ n'ont pas été décrites.

À l'aide de données accessibles au public sur les cas allemands rapportés quotidiennement, les modèles de croissance exponentielle pour les infections et le $R_{t}$ ont été estimés et étudiés en ce qui concerne les points de changement dans la série chronologique.

Ehrhardt (2020) (25) Cette étude visait à évaluer la transmission du SRAS-CoV-2 chez Étude écologique Allemagne Fév.-août 2020 les enfants des écoles primaires, des écoles secondaires et des structures de garde d'enfants du Bade-Wurtemberg (Allemagne) après la réouverture des écoles en mai 2020. Les mesures de PCI comprenaient : réduction de la taille des classes, désinfection, hygiène des mains et interdiction des sports et de la musique dans les écoles primaires et secondaires.

Une courbe épidémique a été utilisée pour montrer de nouveaux cas quotidiens après la réouverture des écoles.
Aucun effet significatif n'a été observé sur les infections de COVID-19 qui pourraient être attribuées à la fermeture d'écoles et de garderies.
La transmission d'enfant à enfant dans les écoles était faible.

L'étude a estimé qu'un cas secondaire provenait de 25 jours d'école infectieuse (jours passés à l'école pendant la période infectieuse).

Les réouvertures à l'école n'étaient pas associées à un changement dans la transmission du SRAS-CoV-2. 